\title{
Multimodal Ensemble Deep Learning to Predict Disruptive Behavior Disorders in Children
}

OPEN ACCESS

Edited by:

Itir Onal Ertugrul,

Tilburg University, Netherlands

Reviewed by:

Fahad Saeed

Florida International University,

United States

Rajat Mani Thomas,

Academic Medical Center,

Netherlands

Yunyan Zhang,

University of Calgary, Canada

*Correspondence:

K. Krishnamurthy

kkrishna@mst.edu

Received: 16 July 2021 Accepted: 15 October 2021 Published: 24 November 2021

Citation:

Menon SS and Krishnamurthy $K$ (2021) Multimodal Ensemble Deep Learning to Predict Disruptive Behavior Disorders in Children. Front. Neuroinform. 15:742807. doi: 10.3389/fninf.2021.742807

\section{Sreevalsan S. Menon and K. Krishnamurthy*}

Department of Mechanical and Aerospace Engineering, Missouri University of Science and Technology, Rolla, MO, United States

Oppositional defiant disorder and conduct disorder, collectively referred to as disruptive behavior disorders (DBDs), are prevalent psychiatric disorders in children. Early diagnosis of DBDs is crucial because they can increase the risks of other mental health and substance use disorders without appropriate psychosocial interventions and treatment. However, diagnosing DBDs is challenging as they are often comorbid with other disorders, such as attention-deficit/hyperactivity disorder, anxiety, and depression. In this study, a multimodal ensemble three-dimensional convolutional neural network (3D CNN) deep learning model was used to classify children with DBDs and typically developing children. The study participants included 419 females and 681 males, aged 108-131 months who were enrolled in the Adolescent Brain Cognitive Development Study. Children were grouped based on the presence of DBDs $(n=550)$ and typically developing ( $n=550)$; assessments were based on the scores from the Child Behavior Checklist and on the Schedule for Affective Disorders and Schizophrenia for School-age Children-Present and Lifetime version for DSM-5. The diffusion, structural, and restingstate functional magnetic resonance imaging (rs-fMRI) data were used as input data to the 3D CNN. The model achieved $72 \%$ accuracy in classifying children with DBDs with $70 \%$ sensitivity, $72 \%$ specificity, and an F1-score of 70 . In addition, the discriminative power of the classifier was investigated by identifying the cortical and subcortical regions primarily involved in the prediction of DBDs using a gradient-weighted class activation mapping method. The classification results were compared with those obtained using the three neuroimaging modalities individually, and a connectome-based graph CNN and a multi-scale recurrent neural network using only the rs-fMRI data.

Keywords: deep learning, disruptive behavior disorders, multimodal ensemble learning, neuroimaging, 3D CNN

\section{INTRODUCTION}

Magnetic resonance imaging (MRI) is a powerful noninvasive neuroimaging tool that can reveal anatomical features and neuronal activities inside a brain. MRI data is widely used to study cognitive development, pathologies, and psychiatric disorders. Diffusion MRI (dMRI) can reveal information about the microstructures, fiber connections, and anatomical connectivities within the brain, and the static anatomical images acquired using structural MRI (sMRI) provide information about the gross anatomical structures in the brain. Dynamic activities inside the brain are measured using functional MRI (fMRI), which is used to identify brain activities in the absence of a task (resting-state fMRI; rs-fMRI) or during a task (task fMRI; tfMRI). 
Disruptive behavior disorders (DBDs) include oppositional defiant disorder (ODD; a pattern of angry/irritable mood, argumentative/defiant behavior, or vindictiveness lasting at least 6 months) and conduct disorder (CD; behavior in which the basic rights of others or major age-appropriate societal norms or rules are violated; American Psychiatric Association, 2013). They are prevalent in children and the most common reasons for referring children to mental health services (Hawes et al., 2020). ODD is estimated to occur in $2-16 \%$ of youth, depending on the population being studied and the method for diagnosis, and $\mathrm{CD}$, which is more prevalent among younger males, rates range from 6 to 9\% (SAMHSA, 2011). DBDs are associated with increased risk for other mental health and substance use disorders (Nock et al., 2006), and are predictors of poor mental health conditions (Scarmeas et al., 2007). These disorders can cause substantial economic losses for society in terms of service utilization (Rivenbark et al., 2018). Therefore, early diagnosis of DBDs is crucial to lower the risk for subsequent disorders with appropriate psychosocial interventions and treatment. However, DBDs are challenging to diagnose as they are often comorbid with other disorders, such as attention-deficit/hyperactivity disorder, anxiety, and depression (Allen et al., 2020).

Machine learning concepts are now receiving increased attention for analysis and prediction in neuroimaging applications. Traditional machine learning techniques require hand-engineered feature selection, which are time-consuming and prone to bias due to manual feature selection. Deep learning is a recent development in machine learning that overcomes the issues associated with hand-engineering and requisite domain expertise for feature selection. Deep learning is a representation learning in which raw data are fed into a learning algorithm that decomposes it into multiple levels of complex nonlinear representative patterns of the input data (LeCun et al., 2015). The burgeoning wide applications of deep learning models can be attributed to the implementation of a convolutional neural network $(\mathrm{CNN})$ because it cut the second-best error rate for image classification by nearly half at the ImageNet Large-Scale Visual Recognition Challenge in 2012 (Krizhevsky et al., 2012). With the advent of parallel computing and graphics processing units, deep representation learning was successfully implemented in numerous areas, such as image processing and analysis tasks, natural language processing, speech recognition, and data synthesis and analysis (LeCun et al., 2015). A large number of medical image analyses now focus on applying deep learning methods to extract features from raw data for further analysis and interpretation (Lundervold and Lundervold, 2019).

CNNs inspired by visual neuroscience are one of the widely used deep learning architectures. A typical CNN includes a convolutional layer, pooling layer, and fully connected layer. The convolutional layer consists of filters/kernels of fixed size that strides with a partial overlap through the input and generates feature maps that are locally weighted sum of input features. Each filter in a convolutional layer looks for the same pattern in different parts of the input, and outputs a unique feature map. The convolution filter thus looks for highly correlated local motifs that can occur at any location in the input (LeCun et al., 2015). The feature maps in the convolution layer are then passed through nonlinear activation functions, such as the rectified linear unit (ReLu) (O'Shea and Hoydis, 2017). The output from one or more convolution layers is then pooled in a pooling layer that merges similar features. Pooling filters output the average or maximum value inside the filter grid and impart translational invariance, for inputs with minor shifts and distortions in rows or columns, to the activation map. Typically, several convolutional and pooling layers are stacked in a $\mathrm{CNN}$, and they are followed by a fully connected layer. The fully connected layer usually connects to an output layer, which could be a softmax function for classification tasks or a linear or support vector machine for regression tasks. CNNs learn in a hierarchical fashion from lowlevel features, such as edges (similar to primary visual cortex), to high-level features, such as shapes (identical to the secondary visual cortex), in deep layers similar to the hierarchical structure in a human visual cortex (Hubel and Wiesel, 1962). Brainnet CNN (Kawahara et al., 2017) is an earlier developed connectomebased graph CNN which is composed of edge-to-edge, edgeto-node, and node-to-graph convolutional filters that leverage the topological locality of brain networks as opposed to local spatial filtering.

CNNs are often considered "black boxes" that perform classifications without explanations on what a model learned or which part of an input was responsible for the classification. One primary goal of machine learning in neuroimaging is to reveal neuromarkers that are indicative of brain health, and diseases and disorders (Khosla et al., 2019). To address these issues, visualization techniques can be utilized to discover discriminative features learned by a CNN model. Class activation mapping (CAM) is a technique to obtain visual explanations of the input regions that a CNN emphasized in its classification (Zhou et al., 2016; Selvaraju et al., 2017) by calculating the derivative of the CNN classification function estimated via back-propagation with respect to the input data. Gradient CAM (Grad-CAM) and Grad$\mathrm{CAM}++$ are two improved versions of CAMs because they can be applied to a wide variety of networks without global average pooling and retraining, and they reveal the discriminative regions in any CNN architecture (Selvaraju et al., 2017; Chattopadhyay et al., 2018). The three CAM techniques were compared in one study on classifying multiple sclerosis types, and it was shown that Grad-CAM outperformed CAM and Grad-CAM++ (Zhang et al., 2021).

A multi-scale recurrent neural network (MsRNN) is another deep learning-based framework that can directly work on the dynamic spatiotemporal fluctuations in the brain activity measured using rs-fMRI time courses for identifying brain disorders (Yan et al., 2019). While the CNN models, deep in space, can be used as an "encoder" for obtaining correlations between brain regions, recurrent neural network (RNN) models, deep in time, can be utilized in sequence classification (Yan et al., 2019). A simple RNN consists of input, hidden and output layers, and it processes the input sequentially with respect to time. The distinguishing feature of RNNs is that the output from a layer is used as input for the layer itself, thereby forming a feedback loop. This allows the RNN to have a history of the sequence elements that can be used to predict the upcoming sequence elements. 
Several studies in machine learning showed that the performance of the learning algorithm can be improved using ensemble learning, which is an algorithm-independent machine learning strategy (Opelt et al., 2004; Khosla et al., 2019). Moreover, brain abnormalities are heterogeneous and cause alterations in functional connectivity and structural changes (McLaughlin et al., 2019). Studies have found abnormal brain activities in children with DBDs using dMRI (Hummer et al., 2015), sMRI (Wallace et al., 2014; Hummer et al., 2015; Waller et al., 2020), tfMRI (Rubia et al., 2009; Hawes et al., 2020), and rs-fMRI ( $\mathrm{Lu}$ et al., 2015; Werhahn et al., 2020). Therefore, there is significant motivation to take advantage of complementary information on various aspects of neuropathology. This study addresses a knowledge gap in the availability of multimodal tools for studying brain abnormalities using different neuroimaging modalities.

In this study, a 3D CNN ensemble deep learning model framework with multimodal neuroimaging data was exploited to identify children with DBDs. The dMRI, sMRI, and rs-fMRI data from a subsample of children enrolled in the Adolescent Brain Cognitive Development (ABCD) Study (Casey et al., 2018) were used as the input data. Furthermore, the brain regions involved in classifying children with DBDs were identified utilizing GradCAM that illustrated the discrimination power of the classifier and the ability to identify neuroimaging phenotypes for DBDs. To assess improvements offered by the ensemble learning, the results were compared with those obtained using the three neuroimaging modalities individually; they were also compared with those obtained using two other readily available deeplearning frameworks, Brainnet CNN and an MsRNN, model with rs-fMRI data. We hypothesized that the classification performance of the ensemble deep learning model will be significantly better than the single modality models.

\section{MATERIALS AND METHODS}

\subsection{Dataset}

Data used in this study came from the ABCD Study that recruited 11,878 children ( $48 \%$ female; $52 \%$ male) between 108 and 120 months of age across 21 sites in the United States. A detailed description of the recruitment, demographics, physical health, and mental assessment and imaging protocols for the study can be found elsewhere (Barch et al., 2018; Casey et al., 2018; Garavan et al., 2018). The baseline ABCD Study data used in this study were from the annual 2.0.1 data release and can be downloaded from the National Institute of Mental Health (NIMH) Data Archive $^{1}$. The data is available to qualified researchers at no cost after their NIMH Data Archive Data Use Certification has been approved. Children with DBDs were identified using the Child Behavior Checklist (CBCL) and the Schedule for Affective Disorders and Schizophrenia for School-Age Children-Present and Lifetime version for DSM-5 (K-SADS-PL) (Hawes et al., 2020). Specifically, the criterion included children who: (i) scored at or above the borderline clinical range (i.e., T-scores $\geq 67$ ) on either the CBCL DSM-oriented conduct problems subscale

$\overline{{ }^{1} \text { https://dx.doi.org/10.15154/1504041 }}$ or oppositional defiant problems subscale; or (ii) received a K-SADS-PL conduct disorder or oppositional defiant disorder diagnosis. Based on this criterion, there were 1,100 children with minimally preprocessed data with all three neuroimaging modalities, i.e., dMRI, sMRI, and rs-fMRI.

\subsection{Preprocessing of ABCD Study Minimally Preprocessed Data}

DTI data were preprocessed using FSL (FMRIB's Software Library $^{2}$ ) scripts, which were used to perform nonlinear registration and projection onto an alignment-invariant tract representation of fractional anisotropy (FA) and mean diffusivity (MD). First, diffusion tensor models were fit at each voxel by using FMRIB's Diffusion Toolbox (FDT, part of FSL). Second, brain extraction was performed using the brain extraction tool (BET) (Smith, 2002). Third, nonlinear registration was done, thereby aligning all FA and MD images to a FMRIB58_FA standard-space image, which has a $1 \times 1 \times 1 \mathrm{~mm}$ resolution, as the target. Finally, all images were resampled back to the $2 \times 2 \times 2$ mm FSL default MNI152 standard-space template resolution. Figure $\mathbf{S 1}$ shows an example DTI image.

The sMRI T1-weighted images were preprocessed mainly using the FSL software. First, extraction of the brain tissue from the skull was performed by using BET. Second, registration to standard space images was carried out using FLIRT (Jenkinson and Smith, 2001; Jenkinson et al., 2002). Third, registration from high-resolution structural to the FSL default MNI152 standard space was then further refined using FNIRT nonlinear registration (Andersson et al., 2007a,b). Finally, the FMRIB's Automated Segmentation Tool (FAST) (Zhang, 2001) was used to segment the brain 3D-image into three different tissue types: (i) gray matter; (ii) white matter; and (iii) cerebrospinal fluid (CSF). Figure S2 shows an example sMRI image.

The rs-fMRI data preprocessing was carried out using FEAT (FMRI Expert Analysis Tool) Version 6.00, a part of FSL. Registration to high-resolution structural and the FSL default MNI152 standard space images was carried out using FLIRT. Registration from high-resolution structural to standardspace was further refined using FNIRT nonlinear registration. Additionally, the following pre-statistics processing was applied: (i) motion correction using MCFLIRT (Jenkinson et al., 2002); (ii) non-brain removal using BET; (iii) spatial smoothing using a Gaussian kernel of FWHM 8.0 mm; (iv) grand-mean intensity normalization of the entire $4 \mathrm{D}$ dataset by a single multiplicative factor, which was done by default in all the fMRI software packages to ensure each image scan had roughly the same mean; and (v) high-pass temporal filtering (Gaussian-weighted leastsquares straight-line fitting, with sigma $=50.0 \mathrm{~s}$ ). The Pearson seed-based correlation values were calculated for four regions of interest, namely posterior and anterior cingulate cortex (PCC and ACC), medial prefrontal cortex (mPFC) and ventral caudate, which are known to be affected in children with DBDs (Alegria et al., 2016). Figure $\mathbf{S} 3$ shows an example rs-fMRI image for the ACC.

${ }^{2}$ www.fmrib.ox.ac.uk/fsl 
TABLE 1 | Demographic and clinical characteristics of the study pool.

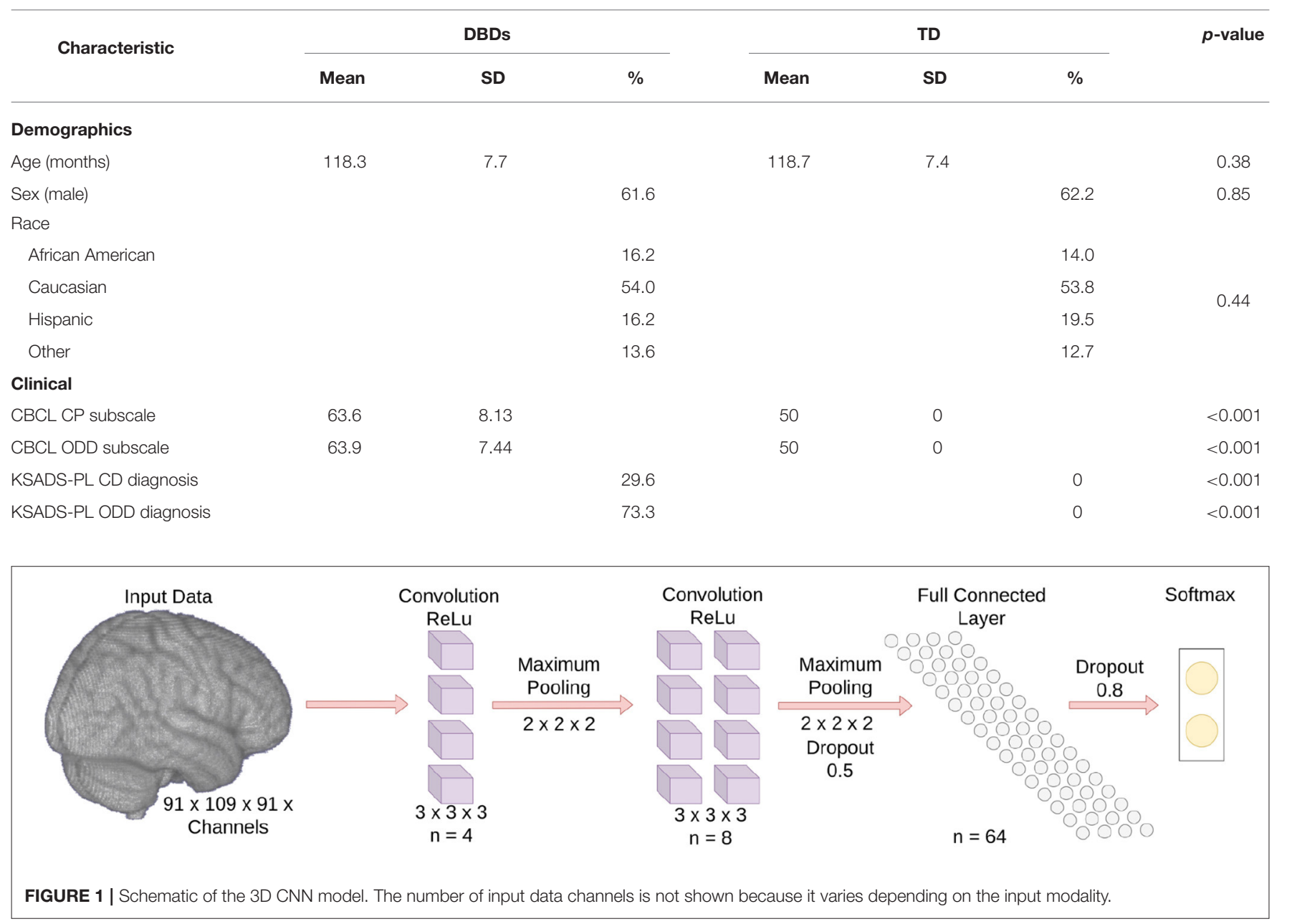

Children were removed from the study pool following preprocessing due to high motion (framewise displacement $>$ $0.25 \mathrm{~mm}$ ), misalignment, and registration failures. As a result, the complete preprocessed data were available for 550 children and a matching number of children in age and sex without DBDs (typically developing, TD) were selected from the ABCD Study data as the control group. Table 1 shows the demographic and clinical characteristics of the final study pool. Descriptive statistics show that the groups were equivalent on demographic variables and significantly different on clinical scores.

\subsection{Ensemble Learning}

Three multichannel 3D CNNs whose inputs were dMRI, sMRI, and rs-fMRI, respectively, were trained in this study to classify children with DBDs and TD children. The goal for the 3D CNNs was to learn the mapping between input (features related to the microstructural integrity and gross anatomical structure of the brain, and resting-state functional patterns) and label (TD children and children with DBDs), so that the 3D CNNs can predict $\mathrm{DBD}$ in previously unseen children. As shown in Figure 1, each 3D CNN model had two convolution blocks each consisting of a 3D convolutional layer (kernel size 3, stride 1), a ReLU activation layer, and a max-pooling layer (kernel size 2 , stride 2). The number of feature channels were 4 and 8 for the convolution layers, respectively. The last layer was a fully connected layer with 64 neurons to combine the feature vectors, and a dropout layer was used to reduce model overfitting. The output was a softmax classification layer. The input channels for the three 3D CNN models were as follows: (i) dMRI model-two channels for FA and MD values; (ii) sMRI model-three channels for gray matter, white matter, and CSF; and (iii) rs-fMRI modelfour channels for Pearson correlation of seed regions ACC, PCC, $\mathrm{mPFC}$, and ventral caudate. The three models were combined in an ensemble learning strategy that gave equal weight during maximum voting of the softmax output for classifying children with DBDs and TD children.

The 3D CNN models were trained with mini-batch sizes of 32 with early stopping conditioned on validation accuracy. The binary cross-entropy was used as the loss function and the neural network weights were optimized using the Adam optimizer. The learning rate and gradient decay were set to 0.001 and 0.9 , respectively. The squared gradient decay, epsilon, and maximum epochs were set to $0.9,0.001$, and 50 , respectively. No attempt was made to optimize the aforementioned parameters. 

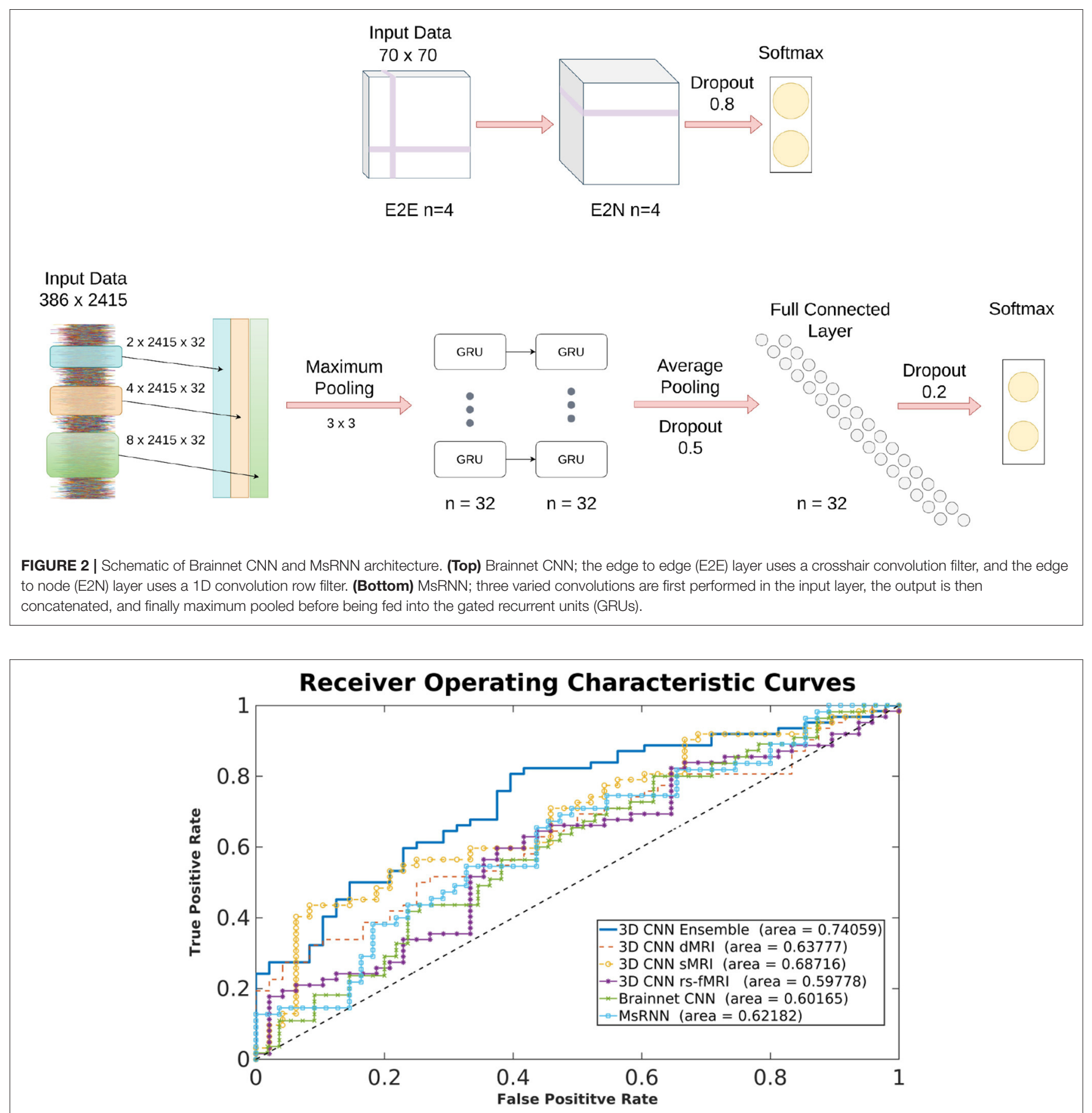

FIGURE 3 | Typical receiver operating characteristic curves for different models.

To ensure that all 3D CNN models relied on information from the same voxels, the FSL default MNI152 standard-space mask was applied to the voxel-level data before feeding into the $3 \mathrm{D}$ CNN model (Khosla et al., 2019). This step removed voxels that may have emerged outside the standard brain template because the preprocessing transformation matrix does not create the exact brain boundary.

\subsection{Brainnet CNN}

As shown in Figure 2, input to the Brainnet CNN was a functional connectivity matrix obtained using timeseries extracted from 70 resting-state networks, which were identified using publicly available 70-component independent component analysis maps (Smith et al., 2009). The blood oxygenation leveldependent (BOLD) timeseries were extracted from the 70 brain 
TABLE 2 | Classification performance in percentage.

\begin{tabular}{|c|c|c|c|c|c|c|c|}
\hline \multirow{2}{*}{ Method } & \multirow{2}{*}{ Modality } & \multicolumn{3}{|c|}{ Accuracy } & \multirow{2}{*}{$\begin{array}{l}\text { Sensitivity } \\
\text { Mean (SD) }\end{array}$} & \multirow{2}{*}{$\begin{array}{l}\text { Specificity } \\
\text { Mean (SD) }\end{array}$} & \multirow{2}{*}{$\begin{array}{l}\text { F1-score } \\
\text { Mean (SD) }\end{array}$} \\
\hline & & Mean (SD) & $p$-value & Cohen's $d$ & & & \\
\hline & $\mathrm{dMRI}$ & $64(2.6)$ & $<0.001$ & 2.20 & $60(16.0)$ & 67 (14.3) & $61(9.7)$ \\
\hline $3 \mathrm{D} C N N$ & sMRI & $66(2.2)$ & $<0.001$ & 1.85 & $64(11.2)$ & 65 (13.2) & $64(6.4)$ \\
\hline MsRNN & rs-fMRI & $62(2.5)$ & $<0.001$ & 2.79 & $56(7.7)$ & $68(8.0)$ & $59(4.4)$ \\
\hline
\end{tabular}

areas by averaging the BOLD signal over all voxels belonging to each brain area. The timeseries were detrended and demeaned, and the data were bandpass filtered in the range of $0.01-0.15 \mathrm{~Hz}$ to improve identification of the resting-state fluctuations (Menon and Krishnamurthy, 2019a). The functional connectivity matrix was obtained using Pearson correlation with normalization to z-scores using the Fisher transformation.

The Brainnet CNN model was implemented in Python by modifying publicly available scripts (Kawahara et al., 2017). The Brainnet CNN model had an edge-to-edge (E2E) layer with four filters, followed by a edge-to-node (E2N) layer with four filters, and finally a dense layer with two neurons. A leaky ReLU nonlinearity with alpha equal to 0.33 was applied to the output of each layer except the last layer, which was a softmax layer. Dropout regularization with a rate of 0.8 was used for the edgeto-node layer and cross-entropy loss was used to optimize the classification model. The models were trained for 1,000 iterations using stochastic gradient descent with a momentum equal to 0.9 . The learning rate was set to 0.001 and a decay of 0.0005 was used for the classification model. No attempt was made to optimize the aforementioned parameters.

\subsection{Multi-Scale Recurrent Neural Network}

Figure 2 shows a schematic of the MsRNN used in this study. The timeseries extracted from 70 resting-state networks that were input into the Brainnet $\mathrm{CNN}$ were also used as the input to an MsRNN. The dynamic correlation connectivity values of 2,415 edges were calculated with a window length of $85 \mathrm{TR}$ and step size of 5 TR (Menon and Krishnamurthy, 2019a). The MsRNN utilized three different scales of $321 \mathrm{D}$ convolutional filters (2 TR, $4 \mathrm{TR}$, and $8 \mathrm{TR}, \mathrm{TR}=0.8 \mathrm{~s}$ ), one concatenation layer, one max-pooling layer of kernel size 3, a two-layer stacked gated recurrent unit GRU with 32 filters which were densely connected in a feed-forward manner, and an averaged layer that integrated the whole sequence followed by a dense layer of 32 neurons before the softmax classification layer. Dropout layers were used before and after the dense neurons with 50 and 20\% dropout, respectively, and L1 and L2 regularization of 0.01 was used to avoid overfitting the data. The MsRNN was trained in Python following Yan et al. (2019) with a mini-batch size of 32, and included early stopping conditioned on validation accuracy and a learning rate of 0.001 . The binary cross-entropy was used as the loss function, and the neural network weights were optimized using the Adam optimizer. No attempt was made to optimize the aforementioned parameters.

\section{RESULTS}

\subsection{Experiments}

To test the efficacy of the multimodal data ensemble, a ten-fold cross-validation (CV) strategy with maximum voting was investigated. The Grad-CAM method was applied to the predicted output, and the results for all the children with DBDs and TD children were averaged to delineate the global trends of the important regions involved in the classification. To benchmark the performance of the ensemble learning approach, the results were compared to those obtained from: (i) the three 3D CNN models used in the ensemble learning considered individually; (ii) Brainnet CNN; and (iii) MsRNN model.

\subsection{Classification Performance}

Figure 3 shows typical receiver operating characteristic curves and Table 2 shows the performance of the different methods for classifying children with DBDs and TD controls. With 10 -fold cross-validation, the multimodal ensemble model with maximum voting resulted in an average prediction accuracy to $72 \%$. The average prediction accuracies for dMRI, sMRI, and rs-fMRI single modalities were 64,66 , and $66 \%$, respectively, compared to $62 \%$ with the Brainnet CNN and MsRNN. Table 2 also shows the multimodal ensemble model to have higher sensitivity, specificity, and F1-score compared to the other models considered.

Statistical results from two-sample t-tests were used to compare the accuracy of the classification performance of the different models. The higher accuracy of the proposed ensemble model compared to all the other models was significant (highest $p$-value was 0.002 ) with a very large to huge effect size calculated as Cohen's $d$ (Sawilowsky, 2009). Overall, as hypothesized, the classification performance was significantly higher using the ensemble learning model because it utilized complementary information from the three different modalities. The results also indicated the superiority of voxel-based 3D CNN models compared to network-level models, such as Brainnet $\mathrm{CNN}$ and MsRNN. 


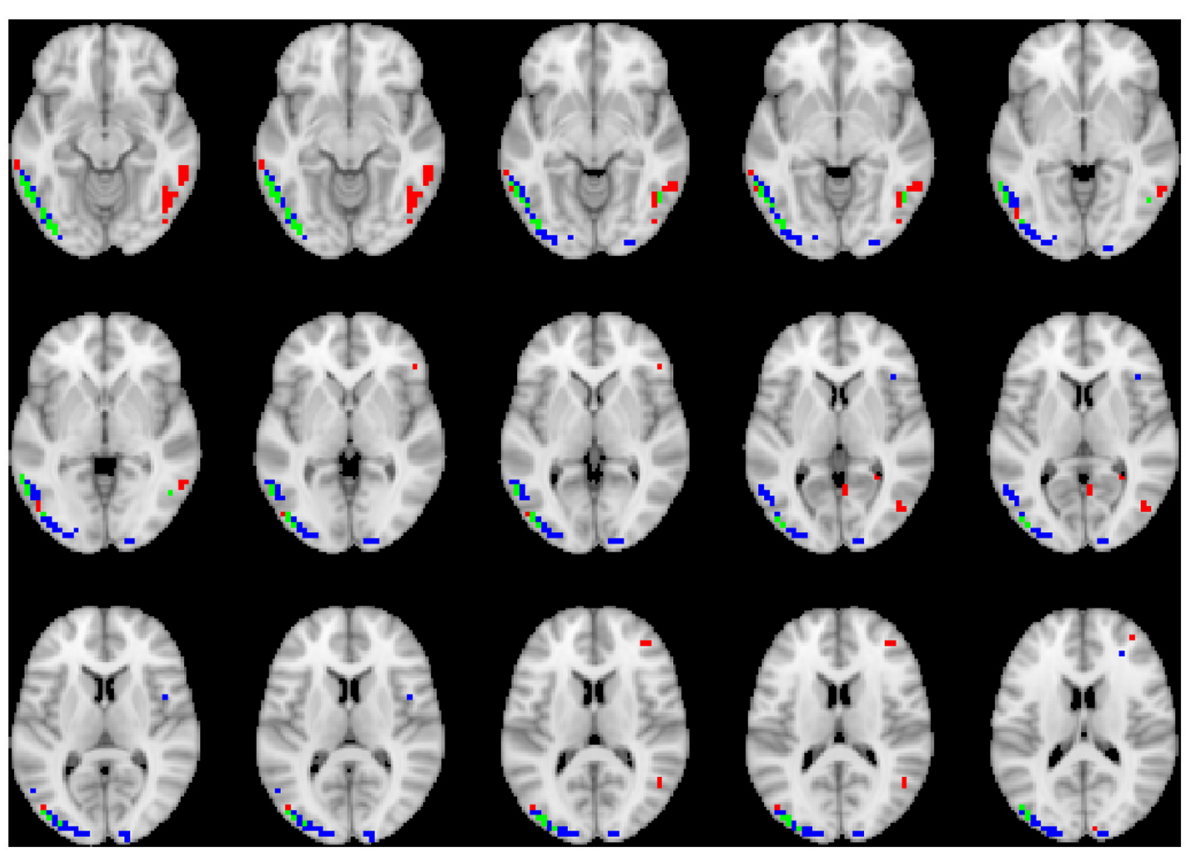

FIGURE 4 | Axial views of voxels primarily contributing to children classification in dMRI image. Green, common to DBD and TD groups; red, DBD group; blue, TD group. The right side of each image corresponds with the right side of the brain.

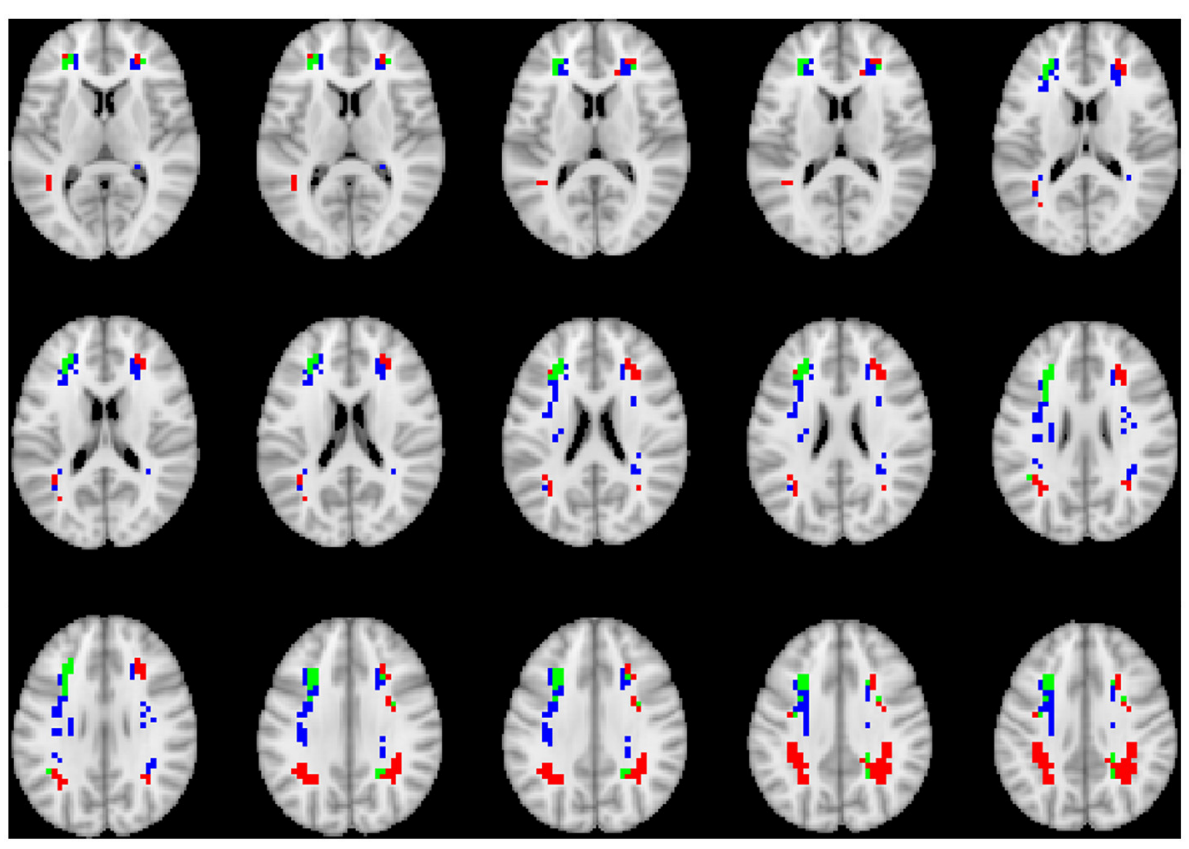

FIGURE 5 | Axial views of voxels primarily contributing to children classification in SMRI image. Green, common to DBD and TD groups; red, DBD group; blue, TD group. The right side of each image corresponds with the right side of the brain.

\subsection{Visualization}

To visualize the brain regions that primarily contributed to children classification, Grad-CAM obtained for children with DBDs and TD children were thresholded at 99 percentile to first identify voxels with high gradient values. The brain regions that primarily contributed toward classification were then identified using the JHU ICBM-DTI-81 white-matter atlas for dMRI image and the AAL atlas for sMRI and rs-fMRI images. Figures 4-6 


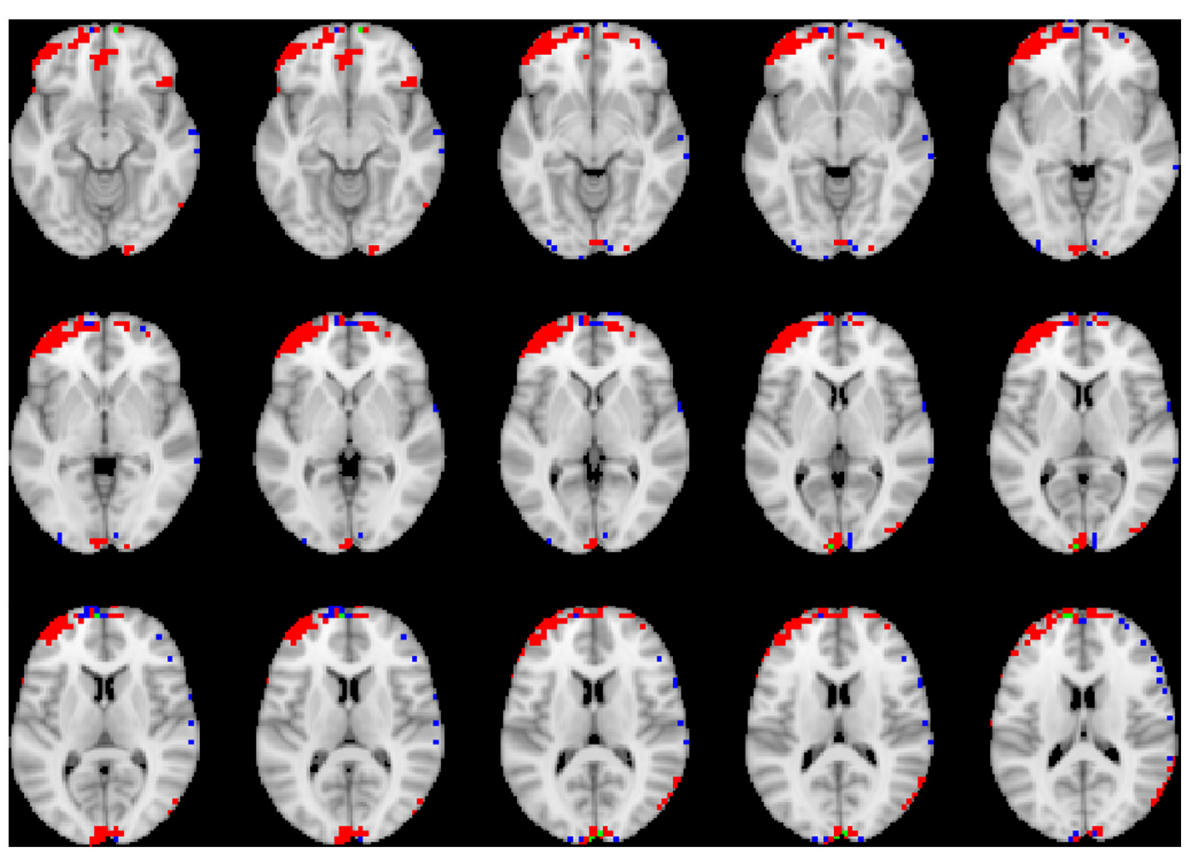

FIGURE 6 | Axial views of voxels primarily contributing to children classification in rs-fMRI image. Green, common to DBD and TD groups; red, DBD group; blue, TD group. The right side of each image corresponds with the right side of the brain.

show axial views of voxels that primarily contributed to the classification of children with DBDs and TD children in dMRI, sMRI, and rs-fMRI images, respectively. Table 3 lists the top five brain regions in the $\mathrm{dMRI}$ images and top ten in the sMRI and rs-fMRI images of children with DBDs and TD children. Some of the regions were common to both groups and are listed in green. On the other hand, regions that were unique to children with DBDs and TD children are listed in red and blue, respectively. These unique regions are of interest because their contributions outweigh the gradient contributions of common regions, hence are highly class discriminative. Further, these unique regions are evidence of abnormalities in children with DBDs because the primarily contributing regions are all different from those in the TD children.

\subsection{D CNN Training Information}

Time for training the 3D CNN model was similar for the three modalities. It typically took around $150 \mathrm{~min}$ per fold on Dell Precision 7,910 and 7,920 Tower workstations with Intel Xeon processors, 128 GB RAM and 1 GB GPU. Figures S4, S5 show a typical training graph and Precision-Recall curves, respectively.

\section{DISCUSSION}

This was the first neuroimaging study to consider the classification of children with DBDs. This is a challenging problem because DBDs are often comorbid with other disorders, such as attention-deficit/hyperactivity disorder, anxiety, and depression. The multimodal ensemble learning approach for diagnosing DBDs with voxel-based $3 \mathrm{D} C N N$ is a novel approach and the accuracy of the ensemble model increased by $6-10 \%$ compared to other models. The maximum voting in the ensemble learning method simulates how clinicians typically make decisions. Given that brain abnormalities are heterogeneous, it is naturally advantageous to utilize information from multimodalities. The maximum voting is the simplest and easiest ensemble method that can be applied to $3 \mathrm{D} \mathrm{CNN}$ models. The maximum voting strategy also ensures that the results are not biased toward any single modality, but will take into account all available information. 3D CNN models, unlike traditional machine learning methods, such as artificial neural networks or support vector machines are well suited to include the spatial relations in the $3 \mathrm{D}$ neuroimaging data, which are known to affect brain functioning. Furthermore, traditional machine learning methods will overfit the data and reduce the validation classification accuracy with high-dimensional 3D neuroimaging training data.

Grad-CAM reveals the discriminant regions in the brain that contributed to the classification of children with DBDs. As shown in Table 3, most of these regions corroborate with results from past studies on abnormal development in children with DBDs. To mention a few, alterations in the white matter integrity of the left inferior fronto-occipital fasciculus were suggested as a potential biomarker of conduct disorder (Graziano et al., 2021). Similarly, superior longitudinal fasciculus areas were shown to have differences in diffusion measurements that suggested poor maturation of structural connections (Hummer et al., 2015) in children with DBDs. Morphological aberrance of frontoparietal 
TABLE 3 | Brain regions primarily contributing to children classification.

\begin{tabular}{lll}
\hline dMRI & sMRI & rs-fMRI \\
\hline Superior longitudinal fasciculus $L$ & Middle frontal gyrus $L$ & Medial superior frontal gyrus $L$ \\
Superior longitudinal fasciculus $L$ - temporal & Superior frontal gyrus $L$ & Superior frontal gyrus, dorsolateral $L$ \\
part & & Rectus gyrus $L$ (Zhang et al., 2017; Cao et al., 2018) \\
Inferior longitudinal fasciculus $L$ & Angular gyrus $L$ & Rectus gyrus $R$ \\
Superior longitudinal fasciculus $R$ & Precentral gyrus $L$ &
\end{tabular}

(Haney-Caron et al., 2014; Hummer et al.,

2015; Lindner et al., 2016; Sarkar et al., 2016;

Puzzo et al., 2018)

Superior longitudinal fasciculus $\mathrm{R}$ - temporal

part

Inferior fronto-occipital fasciculus $\mathrm{L}$

(Haney-Caron et al., 2014; Lindner et al., 2016;

Graziano et al., 2021)

Forceps major (Lindner et al., 2016)

\author{
Superior frontal gyrus $\mathrm{R} \quad$ Middle frontal gyrus L (Lu et al., 2015; Zhang et al., \\ 2015; Cao et al., 2018) \\ Middle frontal gyrus R (Huebner et al., 2008; \\ Medial orbital superior frontal gyrus $L$
}

Fairchild et al., 2015)

Postcentral gyrus L (Hyatt et al., 2012)

Middle temporal gyrus L (Huebner et al., 2008; Fairchild et al., 2011)

Inferior parietal lobule L (Wallace et al., 2014)

Middle occipital gyrus R (Huebner et al., 2008)

Inferior frontal gyrus, triangular part $\mathrm{L}$ (Huebner et al., 2008; Fairchild et al., 2011; Hyatt et al., 2012)

Inferior frontal gyrus, opercular part L (Huebner

et al., 2008; Fairchild et al., 2011; Hyatt et al., 2012)

Inferior frontal gyrus, opercular part R (Hyatt et al., 2012; Fairchild et al., 2013)

Precentral gyrus R (Hyatt et al., 2012; Fairchild

et al., 2013; Jiang et al., 2015)

Hippocampus R (Waller et al., 2020)
Medial superior frontal gyrus R (Zhang et al., 2017; Cao et al., 2018)

Inferior temporal gyrus L (Zhang et al., 2015; Cao et al., 2018; Werhahn et al., 2020)

Temporal pole superior temporal gyrus $L$ (Cao et al., 2018)

Inferior parietal lobule L (Zhang et al., 2015)

Postcentral gyrus R (Lu et al., 2015; Cao et al., 2018, 2019; Lu F. et al., 2020; Werhahn et al., 2020)

Supramarginal gyrus R (Zhang et al., 2015, 2017)

Precentral gyrus R (Lu F. et al., 2020; Werhahn et al., 2020)

Middle temporal gyrus R (Lu et al., 2015; Zhang et al., 2015; Wu et al., 2017)

Middle frontal gyrus R (Zhang et al., 2015; Cao et al., 2019)

Inferior frontal gyrus, opercular part R (Zhang et al., 2015; Cao et al., 2018, 2019)

Inferior frontal gyrus, triangular part R (Zhang et al., 2015; Cao et al., 2018, 2019)

Superior frontal gyrus R (Zhang et al., 2017; Cao et al., 2018)

Past studies corroborating with results obtained are shown in parentheses. Green, common to DBD and TD groups; red, DBD group; blue, TD group; L, left hemisphere; $R$, right hemisphere.

and temporal gyrus areas can lead to disruptive behavior (Huebner et al., 2008; Hyatt et al., 2012; Fairchild et al., 2015) and most of these regions were found to be class discriminative in this study. Functional connectivity alterations have been reported for children with DBDs, and class discriminative regions found using grad-CAM were consistent with many of the reported regions ( $\mathrm{Lu}$ et al., 2015; Werhahn et al., 2020). Functional connectivity values for higher-order cognitive functional regions such as the middle frontal gyrus and superior frontal gyrus were also found to be class discriminative (Lu et al., 2015).

The $72 \%$ average accuracy obtained using the ensemble learning approach is good. Because there are no other studies on classifying children with DBDs to benchmark against, some representative neuroimaging studies using deep learning were reviewed to qualify the multimodal ensemble model performance. El Gazzar et al. (2019) trained a 1D-CNN on a publicly available autism dataset with nearly 2000 participants to classify rs-fMRI images with an accuracy of $\sim 65 \%$. The accuracy improved to $66 \%$ with a 3D CNN (Thomas et al., 2020). Lu H. et al. (2020) obtained an accuracy of $61 \%$ by applying multikernel fuzzy clustering based on an auto-encoder to classify participants with autism spectrum disorder (ASD) using the Autism Brain Imaging Data Exchange (ABIDE) database (nearly 1,050 participants). Using an ensemble approach on ABIDE data, a classification accuracy of $72.3 \%$ was obtained by Khosla et al. (2019). Similar to DBDs, classification of ASD using machine learning methods is also considered challenging because it varies from person-to-person in severity and combination of symptoms. Other studies with a classification accuracy $>70 \%$ are typically in cases where the sample size is $<200$ (see Vieira et al., 2017; Zhang et al., 2020 for an overview). The sample size is an 
important parameter to consider because a negative relationship between accuracy and sample size has been noted (Pulini et al., 2019).

\section{LIMITATIONS AND FUTURE DIRECTIONS}

The robustness of the training models could not be determined by using a leave-site-out cross-validation scheme for the ABCD Study data that was collected from 21 sites with optimized and harmonized measures and procedures (Casey et al., 2018). A kfold cross-validation was used instead because the number of children from each site in the study pool was imbalanced. The number of children with DBDs varied among the different sites, from a low of 3 to a maximum of 113 .

This study investigated the superiority of ensemble learning for classifying brain disorders. The sample size used here was relatively large compared to published works in the field, but it was probably not large enough to take full advantage of CNN models. The models used in this research employed a small number of filters with a shallow architecture, and this decreased the deep learning "black box" depth and not fully fit the training data; and it reduced the computational burden, which is advantageous. A wide range of choices were available to increase the depth of the CNN architecture and optimize the training parameters. Hyperparameter optimization of the $\mathrm{CNN}$ architecture and training parameters were not performed because the focus here was to investigate the superiority of multimodal ensemble learning with simple models. Tuning the hyperparameters using a grid or random search method, for example, is computationally intense. A number of different optimization algorithms have been proposed (Yu and Zhu, 2020); developing an efficient scheme to optimize the hyperparameters is a topic for future investigation.

For the Brainnet CNN and MsRNN, there are unexplored options for selecting an atlas. In this study, a commonly used functional atlas was considered with few filters similar to the multimodal CNN. Correlation does not account for higherorder interactions because it is a first-order transformation (El Gazzar et al., 2019); therefore, different voxel measurements for rs-fMRI, such as entropy (Menon and Krishnamurthy, $2019 \mathrm{~b}$ ) and other connectivity measures can be investigated. The dynamic nature of the functional connectivity was not analyzed due to the increased computational requirements. Also, no comparison was performed with linear models because a voxel-wise analysis of linear models would suffer from the issues of high dimensionality.

Two strategies that may deserve attention are transfer learning and data augmentation (Vieira et al., 2017; Zhang et al., 2020). Transfer learning involves applying features learned from one dataset to tune another similar dataset. Gong et al. (2021) successfully applied transfer learning strategy exploiting big data from UK Biobank (Miller et al., 2016) in the Predictive Analysis Challenge 2019 dataset, achieving first place. Data augmentation is a strategy used in computer vision applications to enlarge the sample size by applying transformations to the data. Data augmentation methods are only now being addressed for medical imaging classification tasks, but further studies are needed for investigating disorders using 3D brain images with voxel-level data (Zhang et al., 2020).

\section{CONCLUSION}

The recent availability of public neuroimaging data, such as the ABCD Study, UK Biobank (Miller et al., 2016), and Child Mind Institute-Healthy Brain Network (Alexander et al., 2017), help researchers to develop novel machine learning techniques for studying brain diseases and disorders. The ensemble method with multiple modalities is ideally suited to model heterogeneity that is typical with brain abnormalities. $3 \mathrm{D}$ CNN together with visualization using grad-CAM is a promising way to identify neuroimaging phenotypes for the diagnosis of DBDs. Future studies are needed to investigate the use of other neuroimaging modalities to better understand the pathophysiology of brain disorders.

\section{DATA AVAILABILITY STATEMENT}

Publicly available datasets were analyzed in this study. This data can be found at: Adolescent Brain Cognitive Development Study (https://dx.doi.org/10.15154/1504041). Custom inhouse MATLAB and Python scripts are made publicly available in GitHub (https://github.com/sreevalsansmenon/ Multimodal_Ensemble).

\section{AUTHOR CONTRIBUTIONS}

SM and KK: conceptualization, methodology, formal analysis, writing-original draft preparation, and writing-review and editing. SM: software. Both authors contributed to the article and approved the submitted version.

\section{ACKNOWLEDGMENTS}

Data used in the preparation of this article were obtained from the Adolescent Brain Cognitive Development (ABCD) Study (https://abcdstudy.org), held in the NIMH Data Archive (NDA). This is a multisite, longitudinal study designed to recruit more than 10,000 children age 108-120 months and follow them over 120 months into early adulthood. The ABCD Study was supported by the National Institutes of Health and additional federal partners under award numbers U01DA041048, U01DA050989, U01DA051016, U01DA041022, U01DA051018, U01DA051037, U01DA050987, U01DA041174, U01DA041106, U01DA041117, U01DA041028, U01DA041134, U01DA050988, U01DA051039, U01DA041156, U01DA041025, U01DA041120, U01DA051038, U01DA041148, U01DA041093, U01DA041089. A full list of supporters is available at https:// abcdstudy.org/federal-partners.html. A listing of participating sites and a complete listing of the study investigators can 
be found at https://abcdstudy.org/scientists/workgroups/. ABCD consortium investigators designed and implemented the study and/or provided data but did not necessarily participate in analysis or writing of this report. The ABCD data used in this report came from https://dx.doi.org/10.15154/1504041.

\section{REFERENCES}

Alegria, A. A., Radua, J., and Rubia, K. (2016). Meta-analysis of fMRI studies of disruptive behavior disorders. Am. J. Psychiatry 173, 1119-1130. doi: 10.1176/appi.ajp.2016.15081089

Alexander, M., Lindsay, E. J., Ai, L., Andreotti, C., Febre, K., Mangone, A., et al. (2017). An open resource for transdiagnostic research in pediatric mental health and learning disorders. Sci. Data 4:170181. doi: 10.1038/sdata. 2017.181

Allen, J. L., Hwang, S., and Huijding, J. (2020). "Disruptive behavior disorders," in The Encyclopedia of Child and Adolescent Development, eds S. Hupp and J. Jewell (John Wiley and Sons Inc.), 1-13. doi: 10.1002/9781119171492.wecad448

American Psychiatric Association (2013). Diagnostic and Statistical Manual of Mental Disorders, 5th Edn. Arlington, VA: American Psychiatric Publishing.

Andersson, J. L., Jenkinson, M., and Smith, S. (2007a). Non-Linear Optimisation. FMRIB technical report TR07JA1, FMRIB Centre, Oxford, UK.

Andersson, J. L., Jenkinson, M., and Smith, S. (2007b). Non-Linear Registration. AKA Spatial Normalisation FMRIB Technical Report TR07JA2. FMRIB Analysis Group of the University of Oxford.

Barch, D. M., Albaugh, M. D., Avenevoli, S., Chang, L., Clark, D. B., Glantz, M. D., et al. (2018). Demographic, physical and mental health assessments in the adolescent brain and cognitive development study: rationale and description. Dev. Cogn. Neurosci. 32, 55-66. doi: 10.1016/j.dcn.2017.10.010

Cao, W., Li, C., Zhang, J., Dong, D., Sun, X., Yao, S., et al. (2019). Regional homogeneity abnormalities in early-onset and adolescent-onset conduct disorder in boys: a resting-state fMRI study. Front. Hum. Neurosci. 13:26. doi: 10.3389/fnhum.2019.00026

Cao, W., Sun, X., Dong, D., Yao, S., and Huang, B. (2018). Sex differences in spontaneous brain activity in adolescents with conduct disorder. Front. Psychol. 9:1598. doi: 10.3389/fpsyg.2018.01598

Casey, B., Cannonier, T., Conley, M. I., Cohen, A. O., Barch, D. M., Heitzeg, M. M., et al. (2018). The adolescent brain cognitive development (ABCD) study: imaging acquisition across 21 sites. Dev. Cogn. Neurosci. 32, 43-54. doi: 10.1016/j.dcn.2018.03.001

Chattopadhyay, A., Sarkar, A., Howlader, P., and Balasubramanian, V. N. (2018). "Grad-CAM++: generalized gradient-based visual explanations for deep convolutional networks," in IEEE Winter Conference on Applications of Computer Vision (WACV) (Lake Tahoe, Nevada), 839-847. doi: 10.1109/WACV.2018.00097

El Gazzar, A., Cerliani, L., van Wingen, G., and Thomas, R. M. (2019). "Simple 1d convolutional networks for resting-state fMRI based classification in autism," in 2019 International Joint Conference on Neural Networks (IJCNN) (Budapest), 1-6. doi: 10.1109/IJCNN.2019.8852002

Fairchild, G., Hagan, C. C., Walsh, N. D., Passamonti, L., Calder, A. J., and Goodyer, I. M. (2013). Brain structure abnormalities in adolescent girls with conduct disorder. J. Child Psychol. Psychiatry All. Discipl. 54, 86-95. doi: 10.1111/j.1469-7610.2012.02617.x

Fairchild, G., Passamonti, L., Hurford, G., Hagan, C. C., Von Dem Hagen, E. A., Van Goozen, S. H., et al. (2011). Brain structure abnormalities in earlyonset and adolescent-onset conduct disorder. Am. J. Psychiatry 168, 624-633. doi: 10.1176/appi.ajp.2010.10081184

Fairchild, G., Toschi, N., Hagan, C. C., Goodyer, I. M., Calder, A. J., and Passamonti, L. (2015). Cortical thickness, surface area, and folding alterations in male youths with conduct disorder and varying levels of callous-unemotional traits. NeuroImage 8, 253-260. doi: 10.1016/j.nicl.2015.04.018

Garavan, H., Bartsch, H., Conway, K., Decastro, A., Goldstein, R., Heeringa, S., et al. (2018). Recruiting the abcd sample: Design considerations and procedures. Dev. Cogn. Neurosci. 32, 16-22. doi: 10.1016/j.dcn.2018.04.004

\section{SUPPLEMENTARY MATERIAL}

The Supplementary Material for this article can be found online at: https://www.frontiersin.org/articles/10.3389/fninf. 2021.742807/full\#supplementary-material

Gong, W., Beckmann, C. F., Vedaldi, A., Smith, S. M., and Peng, H. (2021). Optimising a simple fully convolutional network for accurate brain age prediction in the pac 2019 challenge. Front. Psychiatry 12:658. doi: 10.3389/fpsyt.2021.627996

Graziano, P. A., Garic, D., and Dick, A. S. (2021). Individual differences in white matter of the uncinate fasciculus and inferior fronto-occipital fasciculus: possible early biomarkers for callous-unemotional behaviors in young children with disruptive behavior problems. J. Child Psychol. Psychiatry All. Discipl. doi: 10.1111/jcpp.13444. [Epub ahead of print].

Haney-Caron, E., Caprihan, A., and Stevens, M. C. (2014). DTI-measured white matter abnormalities in adolescents with conduct disorder. J. Psychiatr. Res. 48, 111-120. doi: 10.1016/j.jpsychires.2013.09.015

Hawes, S. W., Waller, R., Byrd, A. L., Bjork, J. M., Dick, A. S., Sutherland, M. T., et al. (2020). Reward processing in children with disruptive behavior disorders and callous-unemotional traits in the abcd study. Am. J. Psychiatry 178, 333-342. doi: 10.1176/appi.ajp.2020.19101092

Hubel, D. H., and Wiesel, T. N. (1962). Receptive fields, binocular interaction, and functional architecture in the cat's visual cortex. J. Physiol. 160, 106-154.2. doi: 10.1113/jphysiol.1962.sp006837

Huebner, T., Vloet, T. D., Marx, I., Konrad, K., Fink, G. R., Herpertz, S. C., et al. (2008). Morphometric brain abnormalities in boys with conduct disorder. J. Am. Acad. Child Adolesc. Psychiatry 47, 540-547. doi: 10.1097/CHI.0b013e3181676545

Hummer, T. A., Wang, Y., Kronenberger, W. G., Dunn, D. W., and Mathews, V. P. (2015). The relationship of brain structure to age and executive functioning in adolescent disruptive behavior disorder. Psychiatry Res. 231, 210-217. doi: 10.1016/j.pscychresns.2014.11.009

Hyatt, C. J., Haney-Caron, E., and Stevens, M. C. (2012). Cortical thickness and folding deficits in conduct-disordered adolescents. Biol. Psychiatry 72, 207-214. doi: 10.1016/j.biopsych.2011.11.017

Jenkinson, M., Bannister, P., Brady, M., and Smith, S. (2002). Improved optimization for the robust and accurate linear registration and motion correction of brain images. NeuroImage 17, 825-841. doi: 10.1006/nimg.2002.1132

Jenkinson, M., and Smith, S. (2001). A global optimisation method for robust affine registration of brain images. Med. Image Anal. 5, 143-156. doi: 10.1016/S1361-8415(01)00036-6

Jiang, Y., Guo, X., Zhang, J., Gao, J., Wang, X., Situ, W., et al. (2015). Abnormalities of cortical structures in adolescent-onset conduct disorder. Psychol. Med. 45, 3467-3479. doi: 10.1017/S0033291715001361

Kawahara, J., Brown, C. J., Miller, S. P., Booth, B. G., Chau, V., Grunau, R. E., et al. (2017). Brainnetcnn: convolutional neural networks for brain networks; towards predicting neurodevelopment. NeuroImage 146, 1038-1049. doi: 10.1016/j.neuroimage.2016.09.046

Khosla, M., Jamison, K., Kuceyeski, A., and Sabuncu, M. R. (2019). Ensemble learning with $3 \mathrm{~d}$ convolutional neural networks for functional connectome-based prediction. NeuroImage 199, 651-662. doi: 10.1016/j.neuroimage.2019.06.012

Krizhevsky, A., Sutskever, I., and Hinton, G. E. (2012). "Imagenet classification with deep convolutional neural networks," in Advances in Neural Information Processing Systems (Lake Tahoe, Nevada), 1097-1105.

LeCun, Y., Bengio, Y., and Hinton, G. (2015). Deep learning. Nature 521, 436-444. doi: $10.1038 /$ nature14539

Lindner, P., Savic, I., Sitnikov, R., Budhiraja, M., Liu, Y., Jokinen, J., et al. (2016). Conduct disorder in females is associated with reduced corpus callosum structural integrity independent of comorbid disorders and exposure to maltreatment. Transl. Psychiatry 6:e714. doi: 10.1038/tp. 2015.216 
Lu, F., Wang, M., Xu, S., Chen, H., Yuan, Z., Luo, L., et al. (2020). Decreased interhemispheric resting-state functional connectivity in male adolescents with conduct disorder. Brain Imaging Behav. 15, 1201-1210. doi: $10.1007 / \mathrm{s} 11682-020-00320-8$

Lu, F.-M., Zhou, J.-S., Zhang, J., Xiang, Y.-T., Zhang, J., Liu, Q., et al. (2015). Functional connectivity estimated from resting-state fmri reveals selective alterations in male adolescents with pure conduct disorder. PLoS ONE 10:e145668. doi: 10.1371/journal.pone.0145668

Lu, H., Liu, S., Wei, H., and Tu, J. (2020). Multi-kernel fuzzy clustering based on auto-encoder for fMRI functional network. Expert Syst. Appl. 159:113513. doi: 10.1016/j.eswa.2020.113513

Lundervold, A. S., and Lundervold, A. (2019). An overview of deep learning in medical imaging focusing on MRI. Zeitsch. Med. Phys. 29, 102-127. doi: 10.1016/j.zemedi.2018.11.002

McLaughlin, K. A., Weissman, D., and Bitrán, D. (2019). Childhood adversity and neural development: a systematic review. Annu. Rev. Dev. Psychol. 1, 277-312. doi: 10.1146/annurev-devpsych-121318-084950

Menon, S. S., and Krishnamurthy, K. (2019a). A comparison of static and dynamic functional connectivities for identifying subjects and biological sex using intrinsic individual brain connectivity. Sci. Rep. 9:5729. doi: 10.1038/s41598-019-42090-4

Menon, S. S., and Krishnamurthy, K. (2019b). A study of brain neuronal and functional complexities estimated using multiscale entropy in healthy young adults. Entropy 21:995. doi: 10.3390/e21100995

Miller, K. L., Alfaro-Almagro, F., Bangerter, N. K., Thomas, D. L., Yacoub, E., Xu, J., et al. (2016). Multimodal population brain imaging in the UK Biobank prospective epidemiological study. Nat. Neurosci. 19, 1523-1536. doi: $10.1038 / \mathrm{nn} .4393$

Nock, M. K., Kazdin, A. E., Hiripi, E., and Kessler, R. C. (2006). Prevalence, subtypes, and correlates of dsm-iv conduct disorder in the national comorbidity survey replication. Psychol. Med. 36, 699-710. doi: $10.1017 /$ S0033291706007082

Opelt, A., Fussenegger, M., Pinz, A., and Auer, P. (2004). "Weak hypotheses and boosting for generic object detection and recognition," in Computer Vision - ECCV 2004 (Berlin; Heidelberg: Springer), 71-84. doi: 10.1007/978-3-540-24671-8 6

O'Shea, T., and Hoydis, J. (2017). An introduction to deep learning for the physical layer. IEEE Trans. Cogn. Commun. Netw. 3, 563-575. doi: 10.1109/TCCN.2017.2758370

Pulini, A., Kerr, W. T., Loo, S. K., and Lenartowicz, A. (2019). Classification accuracy of neuroimaging biomarkers in attention-deficit/hyperactivity disorder: effects of sample size and circular analysis. Biol. Psychiatry 4, 108-120. doi: 10.1016/j.bpsc.2018.06.003

Puzzo, I., Seunarine, K., Sully, K., Darekar, A., Clark, C., Sonuga-Barke, E. J., et al. (2018). Altered white-matter microstructure in conduct disorder is specifically associated with elevated callous-unemotional traits. J. Abnorm. Child Psychol. 46, 1451-1466. doi: 10.1007/s10802-0170375-5

Rivenbark, J. G., Odgers, C. L., Caspi, A., Harrington, H., Hogan, S., Houts, R. M., et al. (2018). The high societal costs of childhood conduct problems: evidence from administrative records up to age 38 in a longitudinal birth cohort. J. Child Psychol. Psychiatry 59, 703-710. doi: 10.1111/jcpp. 12850

Rubia, K., Smith, A. B., Halari, R., Matsukura, F., Mohammad, M., Taylor, E., et al. (2009). Disorder-specific dissociation of orbitofrontal dysfunction in boys with pure conduct disorder during reward and ventrolateral prefrontal dysfunction in boys with pure adhd during sustained attention. Am. J. Psychiatry 166, 83-94. doi: 10.1176/appi.ajp.2008.08020212

SAMHSA (2011). Interventions for Disruptive Behavior Disorders: How to Use the Evidence-Based Practices Kits. Rockville, MD: Center for Mental Health Services; Substance Abuse and Mental Health Services Administration; U.S. Department of Health and Human Services.

Sarkar, S., Dell'Acqua, F., Walsh, S. F., Blackwood, N., Scott, S., Craig, M. C., et al. (2016). A whole-brain investigation of white matter microstructure in adolescents with conduct disorder. PLOS ONE 11:e155475. doi: 10.1371/journal.pone.0155475
Sawilowsky, S. S. (2009). New effect size rules of thumb. J. Modern Appl. Stat. Methods 8, 597-599. doi: 10.22237/jmasm/1257 035100

Scarmeas, N., Brandt, J., Blacker, D., Albert, M., Hadjigeorgiou, G., Dubois, B., et al. (2007). Disruptive behavior as a predictor in Alzheimer disease. Arch. Neurol. 64, 1755-1761. doi: 10.1001/archneur.64. 12.1755

Selvaraju, R. R., Cogswell, M., Das, A., Vedantam, R., Parikh, D., and Batra, D. (2017). "Grad-cam: visual explanations from deep networks via gradient-based localization," in 2017 IEEE International Conference on Computer Vision (ICCV) (Venice), 618-626. doi: 10.1109/ICCV.2017.74

Smith, S. M. (2002). Fast robust automated brain extraction. Hum. Brain Mapp. 17, 143-155. doi: 10.1002/hbm.10062

Smith, S. M., Fox, P. T., Miller, K. L., Glahn, D. C., Fox, P. M., Mackay, C. E., et al. (2009). Correspondence of the brain's functional architecture during activation and rest. Proc. Natl. Acad. Sci. U.S.A. 106, 13040-13045. doi: 10.1073/pnas.0905267106

Thomas, R. M., Gallo, S., Cerliani, L., Zhutovsky, P., El-Gazzar, A., and van Wingen, G. (2020). Classifying autism spectrum disorder using the temporal statistics of resting-state functional mri data with 3D convolutional neural networks. Front. Psychiatry 11:440. doi: 10.3389/fpsyt.2020.00440

Vieira, S., Pinaya, W. H., and Mechelli, A. (2017). Using deep learning to investigate the neuroimaging correlates of psychiatric and neurological disorders: methods and applications. Neurosci. Biobehav. Rev. 74, 58-75. doi: 10.1016/j.neubiorev.2017. 01.002

Wallace, G. L., White, S. F., Robustelli, B., Sinclair, S., Hwang, S., Martin, A., et al. (2014). Cortical and subcortical abnormalities in youths with conduct disorder and elevated callous-unemotional traits. J. Am. Acad. Child Adolesc. Psychiatry 53, 456-465.e1. doi: 10.1016/j.jaac.2013.12.008

Waller, R., Hawes, S. W., Byrd, A. L., Dick, A. S., Sutherland, M. T., Riedel, M. C. et al. (2020). Disruptive behavior problems, callous-unemotional traits, and regional gray matter volume in the adolescent brain and cognitive development study. Biol. Psychiatry 5, 481-489. doi: 10.1016/j.bpsc.2020.01.002

Werhahn, J. E., Mohl, S., Willinger, D., Smigielski, L., Roth, A., Hofstetter, C., et al. (2020). Aggression subtypes relate to distinct resting state functional connectivity in children and adolescents with disruptive behavior. Eur. Child Adolesc. Psychiatry 30, 1237-1249. doi: 10.1007/s00787-020-01601-9

Wu, Q., Zhang, X., Dong, D., Wang, X., and Yao, S. (2017). Altered spontaneous brain activity in adolescent boys with pure conduct disorder revealed by regional homogeneity analysis. Eur. Child Adolesc. Psychiatry 26, 827-837. doi: 10.1007/s00787-017-0953-7

Yan, W., Calhoun, V., Song, M., Cui, Y., Yan, H., Liu, S., et al. (2019). Discriminating schizophrenia using recurrent neural network applied on time courses of multi-site fMRI data. EBioMedicine 47, 543-552. doi: 10.1016/j.ebiom.2019.08.023

Yu, T., and Zhu, H. (2020). Hyper-parameter optimization: a review of algorithms and applications. arXiv [Preprint]. arXiv:2003.05689. Available online at: https://arxiv.org/abs/2003.05689

Zhang, J., Li, B., Gao, J., Shi, H., Wang, X., Jiang, Y., et al. (2015). Impaired frontalbasal ganglia connectivity in male adolescents with conduct disorder. PLoS ONE 10:e145011. doi: 10.1371/journal.pone.0145011

Zhang, J., Zhou, J., Lu, F., Chen, L., Huang, Y., Chen, H., et al. (2017). Investigation of the changes in the power distribution in resting-state brain networks associated with pure conduct disorder. Sci. Rep. 7:5528. doi: 10.1038/s41598-017-05863-3

Zhang, L., Wang, M., Liu, M., and Zhang, D. (2020). A survey on deep learning for neuroimaging-based brain disorder analysis. Front. Neurosci. 14:779. doi: 10.3389/fnins.2020.00779

Zhang, Y., Brady, M., and Smith, S. (2001). Segmentation of brain $\mathrm{mr}$ images through a hidden markov random field model and the expectation-maximization algorithm. IEEE Trans. Med. Imaging 20, 45-57. doi: $10.1109 / 42.906424$

Zhang, Y., Hong, D., McClement, D., Oladosu, O., Pridham, G., and Slaney, G. (2021). Grad-CAM helps interpret the deep learning models trained to classify multiple sclerosis types using clinical brain magnetic resonance 
imaging. J. Neurosci. Methods 353:109098. doi: 10.1016/j.jneumeth.2021.1 09098

Zhou, B., Khosla, A., Lapedriza, A., Oliva, A., and Torralba, A. (2016). "Learning deep features for discriminative localization," in Proceedings of the IEEE Conference on Computer Vision and Pattern Recognition (CVPR) (Las Vegas, Nevada).

Author Disclaimer: This manuscript reflects the views of the authors and may not reflect the opinions or views of the $\mathrm{NIH}$ or $\mathrm{ABCD}$ consortium investigators.

Conflict of Interest: The authors declare that the research was conducted in the absence of any commercial or financial relationships that could be construed as a potential conflict of interest.
Publisher's Note: All claims expressed in this article are solely those of the authors and do not necessarily represent those of their affiliated organizations, or those of the publisher, the editors and the reviewers. Any product that may be evaluated in this article, or claim that may be made by its manufacturer, is not guaranteed or endorsed by the publisher.

Copyright (c) 2021 Menon and Krishnamurthy. This is an open-access article distributed under the terms of the Creative Commons Attribution License (CC BY). The use, distribution or reproduction in other forums is permitted, provided the original author(s) and the copyright owner(s) are credited and that the original publication in this journal is cited, in accordance with accepted academic practice. No use, distribution or reproduction is permitted which does not comply with these terms. 